S. FUKUDA

KODAI MATH. J.

19 (1996), 191-199

\title{
ON BASE POINT FREE THEOREM
}

\author{
SHIGETAKA FUKUdA
}

\section{§1. Introduction}

Let $X$ be a non-singular projective variety with $\operatorname{dim} X=n$ over $C$. And let $\Delta=\sum_{\imath=1}^{s} \Delta_{2}$ be a reduced divisor on $X$ with only simple normal crossings.

Let Strata $(\Delta):=\left\{\Gamma \mid 1 \leqq k \leqq n, 1 \leqq i_{1}<i_{2}<\cdots<i_{k} \leqq s, \quad \Gamma\right.$ is an irreducible component of $\left.\Delta_{\imath_{1}} \cap \Delta_{\imath_{2}} \cap \cdots \cap \Delta_{\imath_{k}} \neq \emptyset\right\}$. A divisor $R$ on $(X, \Delta)$ is, by definition, nef and $\log$ big if $R$ is nef and big and $\left.R\right|_{\Gamma}$ is nef and big for any member $\Gamma$ of $\operatorname{Strata}(\Delta)$ (due to Reid $[8,10.4]$ ).

Note that if $R$ is ample then $R$ is nef and $\log$ big on $(X, \Delta)$.

The purpose of this paper is to prove the following:

MAIN TheOREM ("log effective freeness"). Let $L$ be a nef divisor on $X$ such that $a L-\left(K_{X}+\Delta\right)$ is nef and $\log$ big on $(X, \Delta)$ for some $a \geqq 0$.

Then there exists a natural number $l_{1}$, depending only on $n$ and $a$, such that the complete linear system $\left|l_{1} L\right|$ is base point free.

The paper is organized as follows. In Section 2 we recall known base point free theorems. In Section 3 we give a proof to Reid's "log eventual freedom" theorem in the "smooth" case. In Section 4 we prove the main theorem. In Section 5 we show some results concerning the log canonical divisor.

We follow the notation and terminology of [Utah].

The results of the present paper have been announced in [Résumé].

Acknowledgement. The author would like to thank Prof. S. Mukai for his warm encouragement.

\section{§ 2. Known results concerning base point freeness}

Concerning base point freeness of linear systems on higher dimensional algebraic varieties, the following results are known.

THEOREM 1 ("eventual freedom") (Kawamata-Shokurov [4, Theorem 3-1-1]). If $L$ is a nef divisor on $X$ and $a L-\left(K_{X}+\Delta\right)$ is ample for some $a \geqq 0$, then $|l L|$

Received March 1, 1995. 
is base point free for every $l \gg 0$.

THEOREM 2 ("effective freeness") (Kollár [5]). Notation as in Theorem 1. There exists a natural number $l_{0}$ depending only on $n$ and a such that $\left|l_{0} L\right|$ is base point free.

Proof. For $0<d \ll 1, a L-\left(K_{X}+(1-d) \Delta\right)$ is ample and $(X,(1-d) \Delta)$ is Kawamata-log-terminal. Thus the assertion immediately follows from Kollár $[5,1.1]$.

Q.E.D.

THEOREM 3 ("log eventual freedom") (Reid $[8,10.4]$ ). If $L$ is a nef divisor on $X$ and $a L-\left(K_{X}+\Delta\right)$ is nef and $\log$ big on $(X, \Delta)$ for some $a \geqq 0$, then $|l L|$ is base point free for every $l \gg 0$.

Remark. This theorem is stated in Reid [8] with the idea for its proof. We give the theorem a proof based on this idea in the next section.

This theorem is not valid under the condition that $a L-\left(K_{X}+\Delta\right)$ is not "nef and $\log$ big" on $(X, \Delta)$ but "nef and big" (Zariski [4, Remark 3-1-2], Kawamata [3, Introduction]).

The Main Theorem of this paper is the generalization of Theorem 2 to "log effective freeness".

\section{$\S 3$. Norimatsu Type Vanishing and proof of Theorem 3}

THEOREM 4 (cf. Ein-Lazarsfeld $[1,2.4]$ and Norimatsu [7]) ("Norimatsu Type Vanishing"). Let $R$ be a nef and log big divisor on $(X, \Delta)$. Then

$$
\mathrm{H}^{i}\left(X, \mathcal{O}_{X}\left(K_{X}+\Delta+R\right)\right)=0 \text { for } i>0 .
$$

Proof (similar to the proof of $[1,2.4]$ ). The assertion follows from the following exact sequence by induction on $(n, s)$, by virtue of Kawamata-Viehweg vanishing theorem :

$$
\begin{aligned}
0 \longrightarrow \mathcal{O}_{X}\left(K_{X}+\sum_{j<s} \Delta_{j}+R\right) \longrightarrow \mathcal{O}_{X}\left(K_{X}+\Delta+R\right) \\
\longrightarrow \mathcal{O}_{\Delta_{s}}\left(K_{\Delta_{s}}+\left.\sum_{j<s} \Delta_{j}\right|_{\Delta_{s}}+\left.R\right|_{\Delta_{s}}\right) \longrightarrow 0
\end{aligned}
$$

where $\Delta=\sum_{j=1}^{s} \Delta_{\jmath}$.

Q.E.D.

Now we give Theorem 3 a proof relying on "Norimatsu Type Vanishing" and the method of Kawamata [3].

Proof of Theorem 3 (based on Reid's idea $[8,10.4]$ ). We shall prove the theorem by induction on $n=\operatorname{dim} X$. In the case where $\Delta=0$, this is a Kawamata-Shokurov result [4, Remark 3-1-2]. So we may assume that $\Delta \neq 0$. 
For any $i \in\{1,2, \cdots, s\}$, we consider the following exact sequence:

$$
0 \longrightarrow \mathcal{O}_{X}\left(l L-\Delta_{\imath}\right) \longrightarrow \mathcal{O}_{X}(l L) \longrightarrow \mathcal{O}_{\Delta_{i}}(l L) \longrightarrow 0 .
$$

We assume that $l \geqq a$. Thus $l L-\Delta_{i}-\left(K_{X}+\sum_{\jmath \neq \imath} \Delta_{j}\right)=l L-\left(K_{X}+\Delta\right)$ is nef and $\log$ big on $\left(X, \sum_{j \neq \imath} \Delta_{j}\right)$.

Hence $\mathrm{H}^{1}\left(X, O_{X}\left(l L-\Delta_{2}\right)\right)=0$ from Theorem 4 .

And $\left.a L\right|_{\Delta_{i}}-\left(K_{\Delta_{i}}+\left.\sum_{j \neq \imath} \Delta_{j}\right|_{\Delta_{i}}\right)=\left.\left(a L-\left(K_{X}+\Delta\right)\right)\right|_{\Delta_{i}}$ is nef and log big on $\left(\Delta_{\imath},\left.\sum_{\jmath \neq \imath} \Delta_{j}\right|_{\Delta_{i}}\right)$. Thus by induction hypothesis, Bs $|l L|_{\Delta_{i}} \mid=\emptyset$ for every $l \gg 0$.

Therefore Bs $|l L| \cap \operatorname{Supp} \Delta=\emptyset$ for every $l \gg 0$.

From now, we use the technique of Kawamata [3, Proof of Lemma 2].

Now fix a prime number $p$. We claim that $\mathrm{Bs}\left|p^{m} L\right|=\emptyset$ for $m \gg 0$.

Take a sufficient large natural number $m_{0}$ such that $\operatorname{Bs}\left|p^{m_{0}} L\right| \cap \operatorname{Supp} \Delta=\emptyset$. We assume that $\mathrm{Bs}\left|p^{m_{0}} L\right| \neq \emptyset$.

Take a log resolution $\mu: Y \rightarrow X$ (all relevant divisors $F_{3}$ are smooth and cross normally) such that

(a) $K_{Y}=\mu^{*}\left(K_{X}+\Delta\right)+\Sigma_{j} a_{j} F_{\jmath}$,

(b) $\left|\mu^{*}\left(p^{m_{0}} L\right)\right|=|M|+\sum r_{j} F_{j}(|M|$ is the movable part and is free), and

(c) $\mu^{*}\left(a L-\left(K_{X}+\Delta\right)\right)-\sum_{j} \delta_{j} F_{j}$ is ample (where $\delta_{j} \in \boldsymbol{Q}$ and $0 \leqq \boldsymbol{\delta}_{j} \ll 1$ ).

Let $c:=\min _{r_{j \neq 0}}\left(\left(a_{j}+1-\delta_{j}\right) / r_{j}\right)$.

Here $c>0$, because if $a_{j}=-1$ then $\mu\left(F_{j}\right) \subset \operatorname{Supp} \Delta$ and therefore $r_{j}=0$.

We may assume that the minimum is attained at exactly one value $j=j_{0}$. Note that $F_{\rho_{0}} \cap \mu^{-1}(\Delta)=\emptyset$.

Put $A:=\sum_{j}\left(-c r_{j}+a_{j}-\delta_{j}\right) F_{\jmath}$ and $A^{\prime}:=\left\ulcorner\left(A+F_{\jmath_{0}}+\mu^{-1}(\Delta)\right)\right\rceil=\ulcorner A\urcorner+F_{\jmath_{0}}+\mu^{-1}(\Delta)$ (where $\mu^{-1}(\Delta)$ denotes the set theoretical inverse image with a reduced structure).

Then $A^{\prime} \geqq 0$ and $\mu_{*} A^{\prime}=0$.

We consider a $\boldsymbol{Q}$-divisor $N:=\mu^{*}\left(p^{m} L\right)+A-K_{Y} \equiv c M+\mu^{*}\left(\left(p^{m}-c p^{m_{0}}\right) L\right.$ $\left.-\left(K_{X}+\Delta\right)\right)-\sum \delta_{j} F_{j}$.

If $p^{m}-c p^{m_{0}} \geqq a$, then $N$ is ample and hence $H^{1}\left(Y, \mathcal{O}_{Y}\left(\mu^{*}\left(p^{m} L\right)+\ulcorner A\urcorner\right)\right)=0$.

We consider the following exact sequence:

$$
\begin{aligned}
0 & \longrightarrow \mathcal{O}_{Y}\left(\mu^{*}\left(p^{m} L\right)+\ulcorner A\urcorner\right) \longrightarrow \mathcal{O}_{Y}\left(\mu^{*}\left(p^{m} L\right)+A^{\prime}\right) \\
& \longrightarrow \mathcal{O}_{F_{\gamma_{0}}}\left(\mu^{*}\left(p^{m} L\right)+A^{\prime}\right) \oplus \mathcal{O}_{\mu^{-1}(\Delta)}\left(\mu^{*}\left(p^{m} L\right)+A^{\prime}\right) \longrightarrow 0 .
\end{aligned}
$$

By the Nonvanishing theorem of Shokurov,

$$
\mathrm{H}^{0}\left(F_{J_{0}}, \mathcal{O}_{F_{J_{0}}}\left(\mu^{*}\left(p^{m} L\right)+A^{\prime}\right)\right) \neq 0 \text { for } m \gg 0,
$$

because $\left.p^{m} \mu^{*} L\right|_{F_{\jmath_{0}}}+\left.\left(A+F_{\jmath_{0}}+\mu^{-1}(\Delta)\right)\right|_{F_{\jmath_{0}}}-K_{F_{\jmath_{0}}}=\left.\left(\mu^{*}\left(p^{m} L\right)+A-K_{Y}\right)\right|_{F_{\jmath_{0}}}=\left.N\right|_{F_{\jmath_{0}}}$ is ample.

Thus, for $m \gg 0, \mathrm{Bs}\left|p^{m} L\right|$ does not include $\mu\left(F_{\rho_{0}}\right)$ but $\mathrm{Bs}\left|p^{m_{0}} L\right|$ includes $\mu\left(F_{J_{0}}\right)$. Hence the method of KMM [4, Theorem 3-1-1] implies the theorem.

Q.E.D. 


\section{$\S 4$. Main theorem (log effective freeness)}

MAIN THEOREM ("log effective freeness"). Assume that $L$ is a nef divisor on $X$ and that $a L-\left(K_{X}+\Delta\right)$ is nef and log big an $(X, \Delta)$ for some $a \geqq 0$. Then there exists a natural number $f(n, a)$ (which is $\geqq a$ ), depending only on $n$ and $a$, such that the complete linear system $|f(n, a) L|$ is base point free.

LEMMA 1. Let $L$ be a nef divisor on $X$ such that $a L-\left(K_{X}+\Delta\right)$ is nef and $\log$ big on $(X, \Delta)$ for an integer $a \geqq 0$. Then $\operatorname{Bs}|f(n-1, a) L| \cap \Delta=\emptyset$.

Proof. Put $m_{1}:=f(n-1, a)$. We consider the exact sequence

$$
0 \longrightarrow \mathcal{O}_{X}\left(m_{1} L-\Delta_{\imath}\right) \longrightarrow \mathcal{O}_{X}\left(m_{1} L\right) \longrightarrow \mathcal{O}_{\Delta_{i}}\left(m_{1} L\right) \longrightarrow 0
$$

for all $1 \leqq i \leqq s$. By Theorem $4, H^{1}\left(X, O_{X}\left(m_{1} L-\Delta_{\imath}\right)\right)=0$ because $m_{1} L-\Delta_{i}-$ $\left(K_{X}+\sum_{\jmath \neq \imath} \Delta_{j}\right)$ is nef and log big on $\left(X, \Sigma_{j \neq \imath} \Delta_{j}\right)$. Here Bs $\left|m_{1} L\right|_{\Delta_{i}} \mid=\emptyset$, because $\left.a L\right|_{\Delta_{i}}-\left(K_{\Delta_{i}}+\left.\Sigma_{\jmath \neq \imath} \Delta_{j}\right|_{\Delta_{i}}\right)$ is nef and $\log$ big on $\left(\Delta_{\imath},\left.\Sigma_{\jmath \neq \imath} \Delta_{j}\right|_{\Delta_{i}}\right)$. Thus Bs $\left|m_{1} L\right|$ $\cap \Delta=\emptyset$.

Q.E.D.

Let $g: X \rightarrow S$ be the morphism defined by the linear system $|l L|$ for $l \gg 0$ (Theorem 3). Here there exists a Cartier divisor $L_{S}$ on $S$ such that $L$ is linearly equivalent to $g^{*} L_{S}$, because $\Phi_{|l L|}=\Phi_{|(l+1) L|}=g$ for $l \gg 0$. We may assume that $L=g^{*} L_{S}$.

For any effective divisor $H$ on $X$ which is linearly equivalent to $m L$ for $m \in \boldsymbol{N}$, there exists a divisor $H_{S}$ on $S$ such that $H=g^{*} H_{S}$, because $\Phi_{|l H|}=$ $\Phi_{|(l+1) H|}=g$ for $l \gg 0$.

Because $g_{*} \mathcal{O}_{X}=\mathcal{O}_{S}$, for any Cartier divisor $D$ on $S$ such that $g * D$ is linearly equivalent to $0, \mathcal{O}_{S} \cong g_{*} g^{*} \mathcal{O}_{S}(D)=g_{*} \boldsymbol{O}_{X} \otimes_{\mathcal{O}_{S}} \mathcal{O}_{S}(D)=\mathcal{O}_{S}(D)$.

Thus $|m L|=g^{*}\left|m L_{S}\right|$ for any $m \in N$. $=\emptyset$.

LEMMA 2. Let $m$ be a natural number such that $|m L| \neq \emptyset$ and $\operatorname{Bs}|m L| \cap \Delta$

Let $Z_{S}$ be an irreducible component of $\operatorname{Bs}\left|m L_{S}\right|$ and $\operatorname{set} k=\operatorname{codim}\left(Z_{S}, S\right)$. Then $\mathrm{Bs}\left|(k m+j+a+1) L_{S}\right|$ does not include $Z_{S}$ for $j \geqq 0$ except at most $\operatorname{dim} Z_{S}$ different values of $\mathrm{J}$.

Proof (using Kawamata-Shokurov-Kollár's method [5]). Taking general elements $B_{i} \in|m L|$, put $B=(1 / 2 m) B_{0}+B_{1}+\cdots+B_{k}$. Then $(X, \Delta+B)$ is $\log$ canonical outside $\mathrm{Bs}|m L|$ and $(X, \Delta+B)$ is not log canonical at the points belonging to the inverse image of the generic point of $Z_{S}$ by $g$ (by the argument of Kollár [5, 2.2.1]).

Let $M_{0}:=a L-\left(K_{X}+\Delta\right)+(1 / 2) L$.

Take a $\log$ resolution $f: Y \rightarrow X$ (i.e. $Y$ is smooth and all relevent divisors are smooth and cross normally). Let 


$$
\begin{aligned}
& K_{Y}=f^{*}\left(K_{X}+\Delta\right)+\sum_{\imath} e_{\imath} E_{\imath} \quad\left(e_{\imath} \geqq-1\right) ; \\
& f^{*} B=\sum_{\imath} b_{i} E_{\imath} ; \\
& f^{*} M_{0}=A+\sum_{\imath} p_{i} E_{\imath}\left(A \text { is an ample } \boldsymbol{Q} \text {-divisor and } 0 \leqq p_{i} \ll 1\right) .
\end{aligned}
$$

Put $c:=\min \left\{\left(e_{i}+1-p_{i}\right) / b_{i} \mid Z_{S} \subset g f\left(E_{\imath}\right) ; b_{i}>0\right\}$. By changing the $p_{\imath}$ slightly, we may assume that the minimum is achieved for exactly one index. Let us denote the corresponding divisor by $E_{0}$.

Put $W:=\bigcup_{e_{i}-b_{i}<-1} g f\left(E_{\imath}\right)$.

Claim 1. $0<c<1$.

Proof of Claim 1. We prove $c>0$. If $Z_{S} \subset g f\left(E_{\imath}\right), b_{i}>0$ and $e_{\imath}=-1$, then $f\left(E_{\imath}\right) \subset \Delta$. But this can not occur, because $g^{-1}\left(Z_{S}\right) \cap \Delta=\emptyset$ from $\operatorname{Bs}|m L| \cap \Delta=\emptyset$.

Next we prove $c<1$. Let $z$ be a scheme-theoretic point on $X$ such that $g(z)$ is the generic point of $Z_{S}$. Because $(X, \Delta+B)$ is not log canonical at $z$, $z \in f\left(E_{j}\right)$ and $e_{j}-b_{j}<-1$ for some $j$ Q.E.D.

Claim 2. Put $c^{\prime}:=\max \left\{\left(e_{i}+1\right) / b_{i} \mid e_{i}+1<b_{i}\right\}$. Then $c \leqq c^{\prime}<1$ and $c^{\prime}$ is not affected by $p_{i}^{\prime}$ 's.

Proof of Claim 2. From the proof of Claim 1, $\left(e_{j}+1\right) / b_{j}<1$ and $g f\left(E_{j}\right) \supset Z_{S}$ for some $j$.

Claim 3. If $W$ does not include $g f\left(E_{\imath}\right)$, then $c b_{i}-e_{i}+p_{i}<1$ or $f\left(E_{\imath}\right) \subset \Delta$.

Proof of Claim 3. Here $e_{i}-b_{i} \geqq-1$. If $b_{i} \neq 0$, then $e_{i}-c b_{i} \geqq e_{i}-c^{\prime} b_{i}>-1$ by Claim 2. If $b_{i}=0$ and $e_{\imath}>-1$, then $e_{i}-c b_{i}=e_{\imath}>-1$. If $b_{i}=0$ and $e_{2}=-1$, then $f\left(E_{\imath}\right) \subset \Delta$.

Q.E.D.

Claim 4. $g f\left(E_{0}\right)=Z_{S}$. If $c b_{i}-e_{i}+p_{i} \geqq 1$ and $\imath \neq 0$, then $g f\left(E_{\imath}\right)$ does not include $Z_{S}$.

Proof of Claim 4. If $c b_{i}-e_{i}+p_{i} \geqq 1$, then $g f\left(E_{\imath}\right) \subset W$ or $f\left(E_{\imath}\right) \subset \Delta$, by Claim 3. Because $c b_{0}-e_{0}+p_{0}=1, \quad Z_{S} \subset g f\left(E_{0}\right)$ and $g^{-1}\left(Z_{S}\right) \cap \Delta=\emptyset$, we get $g f\left(E_{0}\right) \subset W$. Here $Z_{S}$ is an irreducible component of $W$. Thus $g f\left(E_{0}\right)=Z_{S}$. If $c b_{i}-e_{i}+p_{i} \geqq 1$ and $Z_{S} \subset g f\left(E_{\imath}\right)$, then $p_{i}<1+e_{\imath}$, because $\Delta$ does not include $f\left(E_{\imath}\right)$. So $b_{i}>0$. Thus $c=\left(e_{i}+1-p_{i}\right) / b_{i}$ by the definition of $c$. Hence $i=0$.

Q.E.D.

Claim 5. If $c b_{i}-e_{i}+p_{i}<0$, then $E_{\imath}$ is f-exceptional.

Proof of Claim 5. Because $c>0$ (Claim 1), $e_{\imath}>0$. Thus $E_{\imath}$ is $f$-exceptional. Q.E.D. 
Proof of Lemma 2 Continued. Let $N_{\jmath}:=(k m+j+a+1) L$ and $N_{\jmath}^{\prime}:=f^{*} N_{\mathrm{J}}$ $-\sum_{\imath \neq 0}\left\llcorner c b_{i}-e_{i}+p_{i}\right\rfloor E_{\imath}$.

We consider the exact sequence

$$
0 \longrightarrow \mathcal{O}_{Y}\left(N_{j}^{\prime}-E_{0}\right) \longrightarrow \mathcal{O}_{Y}\left(N_{j}^{\prime}\right) \longrightarrow \mathcal{O}_{E_{0}}\left(N_{j}^{\prime}\right) \longrightarrow 0 .
$$

Note that

$$
N_{j}^{\prime}-E_{0} \equiv K_{Y}+A+(1-c) f^{*} B+j f^{*} L+\sum_{i \neq 0}\left\{c b_{i}-e_{i}+p_{i}\right\} E_{\imath}
$$

and

$$
\left.N_{j}^{\prime}\right|_{E_{0}} \equiv K_{E_{0}}+\left.\left(A+(1-c) f^{*} B+j f * L\right)\right|_{E_{0}}+\left.\sum_{\imath \neq 0}\left\{c b_{i}-e_{i}+p_{i}\right\} E_{\imath}\right|_{E_{0}} .
$$

By Claim 1, Kawamata-Viehweg vanishing implies $\mathrm{H}^{1}\left(Y, \mathcal{O}_{Y}\left(N_{j}^{\prime}-E_{0}\right)\right)=0$ and $h^{0}\left(E_{0}, \mathcal{O}_{E_{0}}\left(N_{j}^{\prime}\right)\right)=\chi\left(\mathcal{O}_{E_{0}}\left(N_{j}^{\prime}\right)\right)$.

$h^{0}\left(E_{0}, \mathcal{O}_{E_{0}}\left(N_{j}^{\prime}\right)\right)$ is a nonzero polynomial of degree $\operatorname{dim} Z_{S}$ in $j$ for $j \geqq 0$ (by the argument of Kollár [5, p. 600]).

By Claim 4 and Claim 5 , Bs $\left|N_{j}\right|$ does not include $f\left(E_{0}\right)$ for all $j \geqq 0$ except at most $\operatorname{dim} Z_{S}$ different values of $j$.

Noting that $\mathrm{Bs}\left|N_{j}\right|=g^{-1} \mathrm{Bs}\left|(k m+j+a+1) L_{S}\right|$, we end the proof of this lemma.

Q.E.D.

Proof of Main Theorem. By using Lemma 1 and Lemma 2, the same argument as in Kollár [5, 2.3] implies the theorem.

Q.E.D.

Remark. The function $f(n, a)$ is given as follows.

When $n=1$, we put $f(1, a):=2^{2}(1+1) !(a+1)$.

When $n \geqq 2$, we put $f(n, a):=2^{n+1}(n+1) !(a+n) f(n-1, a)$.

Thus $f(n, a)=\prod_{\imath=1}^{n}\left(2^{\imath+1}(i+1) !(a+i)\right)$.

Proof of the Remark Above. When $n=1$, if $\Delta \neq 0$, then $\operatorname{Bs}|2(a+1) L| \cap \Delta=\emptyset$ from the proof of Lemma 1 , because $2(a+1) \geqq a$. If $\Delta=0$, then $|2(a+1) L| \neq \emptyset$ from Kollár $[5,2.4]$. Therefore $B s\left|2^{1}(1+1) ! 2(a+1) L\right|=\emptyset$ from the argument of Kollár [5, 2.3], by using Lemma 2 .

When $n \geqq 2$, if $\Delta \neq 0$, then $\operatorname{Bs}|2(a+n) f(n-1, a) L| \cap \Delta=\emptyset$ from Lemma 1. If $\Delta=0$, then $|2(a+n) L| \neq \emptyset$ from Kollár $[5,2.4]$, thus $|2(a+n) f(n-1, a) L| \neq \emptyset$. Therefore $\mathrm{Bs}\left|2^{n}(n+1) ! 2(a+n) f(n-1, a) L\right|=\emptyset$ from the argument of Kollár [5, $2.3]$, by using Lemma 2 .

Q.E.D.

\section{§5. Appendix} $K_{X}+\Delta$.

In this section we show some results concerning the $\log$ canonical divisor 
Theorem 5. Assume that $L$ is a nef divisor on $X$ and that $a L-\left(K_{X}+\Delta\right)$ is nef and $\log$ big on $(X, \Delta)$ for some $a \geqq 0$. Let $\Gamma$ be a member of $\operatorname{Strata}(\Delta)$ and $d:=\operatorname{dim} \Gamma$. Then $\mathrm{Bs}|m L|$ does not include $\Gamma$ for every $m \geqq 2(d+a)$.

Remark. For the proof, we use the following two propositions.

Proposition 1. Let $R$ be a nef and log big divisor on $(X, \Delta)$ and $\Gamma$ a member of $\operatorname{Strata}(\Delta)$. Let $\pi: \tilde{X} \rightarrow X$ be the blow up with center $\Gamma$ and $E$ the exceptional divisor. Then

$$
\begin{aligned}
& 0 \longrightarrow H^{0}\left(\tilde{X}, \mathcal{O}_{\tilde{X}}\left(\pi^{*}\left(K_{X}+\Delta+R\right)-E\right) \longrightarrow H^{0}\left(\tilde{X}, \mathcal{O}_{\tilde{X}}\left(\pi^{*}\left(K_{X}+\Delta+R\right)\right)\right.\right. \\
& \longrightarrow H^{0}\left(E, \mathcal{O}_{E}\left(\pi^{*}\left(K_{X}+\Delta+R\right)\right)\right) \longrightarrow 0
\end{aligned}
$$

is exact and $\left.\mathcal{O}_{E}\left(\pi *\left(K_{X}+\Delta+R\right)\right) \cong \pi\right|_{E}{ }^{*} \mathcal{O}_{\Gamma}\left(K_{X}+\Delta+R\right)$.

Proof. Let $e:=\operatorname{codim}(\Gamma, X)$. We may assume that $\Gamma \subset \Delta_{1} \cap \Delta_{2} \cap \cdots \cap \Delta_{e}$. Let $\Delta_{\imath}^{\prime}$ be the strict transform of $\Delta_{\imath}$. Thus $\pi *\left(K_{X}+\Delta+R\right)-E=K_{\tilde{X}}-(e-1) E+$ $\pi *\left(\Delta_{1}+\Delta_{2}+\cdots+\Delta_{e}\right)+\sum_{i>e} \Delta_{i}^{\prime}+\pi * R-E=K_{\tilde{X}}-(e-1) E+\Sigma_{\imath} \Delta_{i}^{\prime}+e E+\pi * R-E=K_{\tilde{X}}$ $+\Sigma_{\imath} \Delta_{i}^{\prime}+\pi^{*} R$ and $\pi^{*} R$ is nef and $\log$ big on $\left(\tilde{X}, \Sigma_{\imath} \Delta_{\imath}^{\prime}\right.$ ) (where $\Sigma_{\imath} \Delta_{\imath}^{\prime}$ is with only simple normal crossings). Therefore, by Theorem $4, H^{1}\left(\tilde{X}, \Theta_{\tilde{X}}\left(\pi^{*}\left(K_{X}+\Delta\right.\right.\right.$ $+R)-E))=0$.

Q.E.D.

Remark. Let $\Gamma$ be a member of $\operatorname{Strata}(\Delta)$. We may assume that

$$
\left.\Gamma \subset \Delta_{1} \cap \Delta_{2} \cap \cdots \cap \Delta_{e} \text { (where } e=\operatorname{codim}(\Gamma, X)\right) .
$$

Then $\left.\left(K_{X}+\Delta\right)\right|_{\Gamma}=\left.\left(K_{X}+\Delta_{1}+\Delta_{2}+\cdots+\Delta_{e}+\sum_{i>e} \Delta_{\imath}\right)\right|_{\Gamma}=K_{\Gamma}+\left.\sum_{i>e} \Delta_{\imath}\right|_{\Gamma}$.

Proposition 2. Let $L$ be a nef divisor on $X$ such that $a L-\left(K_{X}+\Delta\right)$ is nef and $\log$ big on $(X, \Delta)$ for some $a \geqq 0$. Let $\Gamma$ be a member of $\operatorname{Strata}(\Delta)$ and $d:=\operatorname{dim} \Gamma$. Then $\mathrm{H}^{0}\left(\Gamma, \mathcal{O}_{\Gamma}(m L)\right) \neq 0$ for every $m \geqq 2(d+a)$.

Proof. We may assume $(*)$ in the remark above. If $m \geqq a$, then

$$
\left.(m L)\right|_{\Gamma}=K_{\Gamma}+\left.\sum_{i>e} \Delta_{\imath}\right|_{\Gamma}+\left.\left(m L-\left(K_{X}+\Delta\right)\right)\right|_{\Gamma},
$$

where $\left.\left(m L-\left(K_{X}+\Delta\right)\right)\right|_{\Gamma}$ is nef and log big on $\left(\Gamma,\left.\Sigma_{i>e} \Delta_{\imath}\right|_{\Gamma}\right)$. Thus $h^{0}(\Gamma$, $\left.\mathcal{O}_{\Gamma}(m L)\right)$ is a polynomial in $m$ for $m \geqq a$, of degree at most $d$, by Theorem 4 . Note that $h^{0}\left(\Gamma, \mathcal{O}_{\Gamma}(m L)\right)$ is a nonzero polynomial by Theorem 3. Thus the same argument as in Kollár [5, 2.4] implies the assertion.

Q.E.D.

Proof of Theorem 5. Let $R:=m L-\left(K_{X}+\Delta\right)$. Then the assertion follows from Proposition 1 and Proposition 2.

Q.E.D.

Theorem 6 (Kollár-Matsuki [6, 4.12.1.2], cf. Iitaka [2, Example 11.6]). Let $f: Y \rightarrow X$ be a birational morphism between non-singular projective varieties. 
Suppose that $K_{Y}=f *\left(K_{X}+\Delta\right)+\sum_{\imath=1}^{t} e_{\imath} E_{\imath}$ and that the union of Supp $\sum_{\imath=1}^{t} E_{\imath}$ and $\operatorname{Exc}(f)$ is a divisor with only simple normal crossings. Then $f\left(E_{\imath}\right) \in \operatorname{Strata}(\Delta)$ for all $i$ such that $e_{2}=-1$.

Remark. We give Theorem 6 an alternative proof, using litaka's Logarithmic Ramification formula [2].

Proof. We assume that $e_{j}=-1$ and that $f\left(E_{j}\right)$ is not a member of $\operatorname{Strata}(\Delta)$, and we shall derive a contradiction.

Note that $f\left(E_{j}\right) \subset \operatorname{Supp}(\Delta)$. Let $\Gamma$ be the minimal member of $\operatorname{Strata}(\Delta)$ which includes $f\left(E_{j}\right)$. We may assume that $\Gamma$ is an irreducible component of $\Delta_{1} \cap \Delta_{2}$ $\cap \cdots \cap \Delta_{e}($ where $e=\operatorname{codim}(\Gamma, X))$. Note that $f\left(E_{j}\right) \subsetneq \Gamma$.

Take a general smooth point $p$ of $f\left(E_{j}\right)$. From now in the proof of this theorem, we consider the problem analytically. There exists a neighborhood $U$ of $p$ in $X$ and a smooth prime divisor $\Delta_{0}$ on $U$, such that $\Delta_{0}, \Delta_{1}, \Delta_{2}, \cdots, \Delta_{e}$ are crossing normally and that $f\left(E_{j}\right) \cap U \subset \Delta_{0}$. We may assume that $\Delta_{l} \cap U=\emptyset$ for any $l>e$.

If we write

$$
K_{f^{-1}(U)}=\left(\left.f\right|_{f^{-1}(U)}\right) *\left(K_{U}+\left.\Delta\right|_{U}+\Delta_{0}\right)+\left(\left.\sum_{\imath=1}^{t} d_{\imath} E_{\imath}\right|_{f^{-1}(U)}-E_{0}\right)
$$

(where $E_{0}$ is the strict transform of $\Delta_{0}$ by $f$ ), then $d_{j} \geqq-1$ from litaka's Logarithmic Ramification Formula ([2, Theorem 11.5]). Therefore $e_{3}>-1$ because $\left(\left.f\right|_{f^{-1}(U)}\right) * \Delta_{0} \geqq\left. E_{\jmath}\right|_{f^{-1}(U)}$. This is a contradiction.

Q.E.D.

Remark. At first the author thought that these two theorems in this section are usefull to get an estimate concerning Main Theorem.

\section{REFERENCES}

[1] L. Ein AND R. LAZARSFELD, Global generation of pluricanonical and adjoint linear series on smooth projective threefolds, J. Amer. Math. Soc., 6 (1993), 875-903.

[2] S. IITAKA, Algebraic Geometry, Grad. Texts in Math., 76, Springer, New York, 1981.

[3] Y. Kawamata, Log canonical models of algebraic 3-folds, Internat. J. Math., 3 (1992), 351-357.

[4] Y. Kawamata, K. Matsuda and K. Matsuki, Introduction to the minimal model problem, Algebraic Geometry, Adv. Stud. Pure Math., 10, 1987, 283-360.

[5] J. Kollár, Effective base point freeness, Math. Ann., 296 (1993), 595-605.

[6] J. Kollár and K. Matsuki, Termination of canonical flips, Astérisque, 211 (1992), 59-68.

[7] Y. NoRIMATSU, Kodaira vanishing theorem and Chern classes for $\partial$-manifolds, Proc. Japan Acad. Ser. A Math. Sci., 54 (1978), 107-108.

[8] M. REID, Commentray by M. Reid. $\$ 10$ of Shokurov's paper "3-fold log flips", Russian Acad. Math. Izv. Sci., 40 (1993), 195-200. 
[Résumé] S. Fukuda, A note on base point free theorem, Proc. Japan Acad. Ser. A Math. Sci., 70 (1994), 173-175.

[Utah] J. Kollár, Flips and abundance for algebraic threefolds, Astérisque, 211 (1992).

Suzuka College of Technology (Suzuka Kōsen)

Shiroko-cho, Suzuka City, Mie Prefecture

510-02, JAPAN 